\title{
Desarrollo Empresarial y Capital Humano en la región Sureste de Coahuila
}

\section{ESJ Social Sciences}

\section{Jesús Francisco Mellado Siller \\ Universidad Autónoma de Coahuila, Mexico}

\section{Edith Reyes Ruiz}

Universidad Autónoma de Coahuila, Mexico

\section{Gladys Aide Garcia Lopez}

Universidad Autónoma de Coahuila, Mexico

Submitted: 20 November 2020

Accepted: 18 December 2020

Published: 31 December 2020

Corresponding author:

Edith Reyes Ruiz

DOI: 10.19044/esj.2020.v16n34p184

(c)

Copyright 2020 Siller J.F.M et. al.

Distributed under Creative Commons BY-NC-ND 4.0 OPEN ACCESS

\section{Resumen}

El presente trabajo tiene como objetivo determinar si hay un efecto significativo entre capital humano y desarrollo empresarial en la región sureste del Estado de Coahuila, México. Se aplicó un instrumento a 243 personas, utilizando el software de AMOS PASS, en el cual se contemplaron los indicadores del capital humano y desarrollo empresarial, de acuerdo con la hipótesis las relaciones encontradas entre las varianzas de los constructos indican que existe un valor estadístico significativo que relaciona a estos constructos. En los resultados se encontró que algunas de las variables fueron: la consideración de pertenencia a la empresa, la percepción de cómo los ve la empresa en función de los conocimientos, y, el reconocimiento de que es el capital humano incrementado por parte de la empresa, demostrando que reconoce este valor como gestor del desarrollo empresarial. De esta manera el capital humano se constituye en uno de los principales factores intervinientes en el desarrollo empresarial.

$\begin{array}{lcr}\text { Palabras } & \text { clave: Capital } & \text { Humano, } \\ \text { Desarrollo } & \text { Empresarial, } & \text { Capital } \\ \text { Intelectual } & & \end{array}$




\title{
Business Development and Human Capital in the southeast region of Coahuila
}

\author{
Dr. Jesús Francisco Mellado Siller, \\ M.PL. Edith Reyes Ruiz, \\ M en Ed. Gladys Aide Garcia Lopez, \\ Universidad Autónoma de Coahuila, Mexico
}

\begin{abstract}
The present work determines if there is a significant effect between human capital and business development in the southeastern region of the State of Coahuila, Mexico. An instrument was applied to 243 people, using the AMOS PASS software, in which the indicators of human capital and business development were considered, according to the hypothesis, the relationships found between the variances of the constructs indicate that there is a significant statistical value that relates to these constructs. In the results it was found that some of the variables were: the consideration of belonging to the company, the perception of how the company sees them based on knowledge, and, the recognition that it is the human capital increased by the company, demonstrating that it recognizes this value as a manager of business development. In this way, human capital becomes one of the main factors involved in business development.
\end{abstract}

Keywords: Human Capital, Business Development, Intellectual Capital

\section{Introduction}

Cuando se habla de capital humano, se habla de constructos: mentales, de inteligencia, de vitalidad, de acciones derivadas, de proacción que solo se observan, en su mayoría por sus manifestaciones, es hasta hace algunos años se ha tomado en cuenta este concepto. Probablemente, facilitaron el desarrollo del concepto, los cambios y evoluciones de lo económico, los tecnológicos sociales, culturales, legales, políticos demográficos y hasta ecológicos. A éstos últimos se les llama Capital Estructural. Y como todos estos cambios intervienen, se han escrito muchos puntos de vista y tratado de determinar el desarrollo de este tipo de capital, sin embargo, siguen muchos problemas sociales, principalmente el bienestar familiar, que debería recibir los efectos directos de los beneficios de la relación capital humano y desarrollo empresarial. Si es que se aplica en el desarrollo de las empresas el Capital 
Humano. El presente trabajo trata determinar si en realidad el capital humano lo están utilizando las empresas del suroeste del estado de Coahuila, en México, para ello se utilizaron las características de lo que se considera Capital Humano, y se está buscando probar la relación con el desarrollo empresarial utilizando herramientas matemáticas estadísticas que nos permitan probar las hipótesis de relación entre estos dos conceptos, los resultados señalan que efectivamente existen las relaciones entre el Capital Humano y los conceptos que conforman el desarrollo empresarial; pero considerando que no todas las empresas fueron encuestadas, al menos las que conformaron la muestra dan información que permiten comprobar que la percepción de los sujetos que laboran en esas industrias están mostrando algunas características que se relacionan con el desarrollo empresarial, lo que tiene como consecuencia que las personas se sientan parte principal de la empresa o como se le conoce "Capital Humano empresarial". (Chiavenato, 2019).

Durante muchos años se consideró que el capital financiero era la fuente principal del desarrollo de las empresas, éste era el motor principal del progreso empresarial, e imprescindible para que las compañías tuvieran éxito, sin embargo, este pensamiento ha cambiado, se considera que es la manera de reclutar y retener una buena fuerza de trabajo es indispensable y también es la llave para la operación de cualquier tipo de negocio, se han encontrado estudios que revelan que el desarrollo de proyectos, sustentado por buenas ideas, el vigor y el entusiasmo del personal que labora, no han sido impedido, por la falta de efectivo o de recursos financieros. Algunas empresas han visto dificultado su crecimiento por no haber mantenido una fuerza de trabajo eficiente y entusiasta: les faltó el capital intelectual.

La ventaja competitiva es necesaria como parte de un componente esencial en las empresas; esta es parte de los elementos que medidos, comparados, principalmente tanto en los organigramas funcionales como en las gestiones económicas de la empresa para que comprendan el valor del capital intelectual en el desarrollo empresarial. Entendiendo con esto, que el capital humano como recurso genera producción y rentabilidad derivando en la competitividad de una organización lucrativa empresarial; incrementando su accionar dentro del mercado, la cual es una de las metas de todo tipo de industria. (Montoya Agudelo \& Boyero Saavedra, 2016).

En Chile, de acuerdo con Brunner \& Gregory, la principal riqueza nacional es el capital humano; en base a los estudios realizados por ellos, el capital intelectual equivale a 8 veces el producto nacional; sin embargo, el stock de capital físico es 2.4 veces el producto de lo que se desprende que en la actualidad los desarrollos empresariales, dependen en gran medida de la educación y de las habilidades desarrolladas por las personas laboralmente activas de su población. Esto evidencia que el uso de conocimientos obtenidos ya sea de manera curricular o extracurricular, refiriéndose a las capacitaciones, 
genera pericias que se utilizan de manera articulada con los trabajos desarrollados en los complejos industriales. Es por esto que en este país se concluye que las sociedades contemporáneas deberán mejorar la gestión del recurso humano. (Brunner \& Gregory., 2003).

Las ventajas potenciales del capital humano, vuelven competitivas a las organizaciones de acuerdo con el enfoque actual de la economía del conocimiento (DeNisi \& Hitt, 2003).

El nuevo escenario del siglo XXI, se está reflejando en la industria del sector turístico en Cartagena de Indias de Colombia; Este reflejo se observa por los rápidos cambios en las empresas turísticas al gestionar el recurso humano para atraer al mejor, elevar la productividad y mantener a los más productivos, debido a esto se ha incrementado una gestión estratégica diferencial, que tiene como base la valoración del conocimiento, y dejando atrás la concepción del hombre "sustituible o permutable" por hombre o imprescindible y se atribuyen al recurso humano. (Passos-Simoncas \& AriasAragonés, 2017; Paredes \& Pinto, 2009).

En México, y en las regiones de otros países se ha mostrado la preocupación por determinar las causas por las cuales crecen en diferentes tasas, y tienen niveles distintos de riqueza. Por ello se han llevado a cabo diseños de modelos teóricos muy funcionales para identificar las múltiples experiencias de crecimiento económico y de acumulación de capital, tanto físico como humano. Se han observado en esos modelos que el capital humano, es factor importante en la economía como ventaja competitiva, y que puede significar en economías de desarrollo, como la de México, el despegue hacia la ruta de mejores estándares de productividad y bienestar. (GermanSoto, Rodríguez, \& Escamilla, 2013).

Otros estudios en México destacan que las empresas que necesitan completar su plantilla, inician un proceso de reclutamiento que ellos consideran adecuado; las firmas determinan el cómo cubrir una vacante, considerando su costo y tiempo de búsqueda, características del puesto, consideración del candidato idóneo, promoción de la vacante, además las firmas buscan a los empleados más calificados, sobre todo aquellos, que los empleadores consideran que pueden generar mayor valor agregado para la empresa. Los individuos, por su parte, buscan empleos que ofrezcan los salarios más altos posibles, así como mejores condiciones laborales en cuanto a seguridad física, estabilidad, desarrollo laboral, y mejores prestaciones, para asegurar la mejor oferta laboral. Ellos invierten en Capital Humano, social e informativo, lo que les permite ser más productivos y tener información del mercado laboral. Esta información del mercado laboral está mejorando debido a las redes sociales. Aunque no es un tema relevante en este trabajo no se puede negar la fuerte intervención de este fenómeno social, es lo que se conoce 
como la cuarta transformación. (Petrongolo \& Pissarides , 2001)(Charles, Torres, \& Castro, 2018).

\section{Metodología:}

El presente trabajo es una investigación de tipo de diagnóstico porque refiere integradamente y con sentido comprensivo los acontecimientos en la sucesividad. Su segunda característica es que es una investigación de hallazgo ya que indica expresamente vínculos o relaciones entre los constructos trabajados que son Capital Humano y Desarrollo Empresarial. Por su orientación funcional es aplicada porque interviene en la realidad completa con el objeto de ejecutar cambios o transformaciones en la misma, es deductiva. Transversal un corte, sección o parte del fenómeno integrado, se mide solo una vez los grupos o variables, no se pretende medir la evolución de éstas.

Dado lo anterior con respecto al tema de relación entre Desarrollo Empresarial y Capital Humano se plantea la siguiente Hipótesis:

\section{H1.- A mejor Capital Humano Mayor Desarrollo Empresarial.}

Los Constructos analizados provienen del Instrumento realizado por el Dr Francisco Treviño Treviño, Director de la Facultad de Ingenieria y Mecanica (FIME) de la Universidad Autónoma de Nuevo León.

Variables: las variables utilizadas para el presente análisis se definen operacionalmente como sigue:

Constructo: Capital Humano

v12.- Personal con Experiencia; es decir, los sujetos que laboran en la empresa tienen experiencia en las labores encomendadas.

V13.- Sueldo igual o Superior en la Industria; es consideración de la persona si su sueldo está de acorde a sus expectativas o si está por encima de ellas.

V14.- Recurso Humano Calificado; definida como si el personal que labora en la empresa tiene la calificación adecuada de desempeño en la misma.

V15.- Capacitación dentro de la empresa; se define, como el reconocimiento por parte del empleado en que su capacitación se está llevando a cabo dentro de la empresa.

V16.- Altos Conocimientos; se define como si el empleado se considera, como persona de alto valor de acuerdo a sus conocimientos de los procesos empresariales.

V17.- Sentido de Pertenencia, se define si el sujeto demuestra alta intensidad de la mercadotecnia interna. 
Constructo: Desarrollo Empresarial.

V23.- Desarrolla Procesos y productos, definidos si las personas que laboran en la empresa observan o participan en los procesos y desarrollo de los productos.

V24.- Procesos Generadores de Tecnología definidos si los empleados observan o participan manejando procesos que actualizan la Tecnología de la empresa.

V25.- Innovador y Dan Seguimiento; se define como la capacidad de reconocer que los productos que se fabrican son innovadores y que, además existe un seguimiento por parte de la empresa en su fabricación.

Muestra: la aplicación del Instrumento se llevó a cabo en la Región sureste del Estado de Coahuila, con un n=243, (Landero, Hernández \& González Ramírez, 2007)

Confiabilidad: la confiabilidad del instrumento se analiza con alfa de Cronbach, encontrando los siguientes resultados. (Cesar, 2004)

Tabla 1.- Estadísticas de fiabilidad

\begin{tabular}{|r|r|}
\hline $\begin{array}{c}\text { Alfa de } \\
\text { Cronbach }\end{array}$ & N de elementos \\
\hline .867 & 43 \\
\hline
\end{tabular}

Fuente: Elaboración Propia

En la tabla 1 se encuentra el valor de $\alpha=.867$ lo que señala que el instrumento tiene validez satisfactoria o que señala que el instrumento, presenta confiabilidad

De acuerdo a los parámetros establecidos por Cronbach (Hair, Black, Babin, \& Anderson, 2010)

\section{Resultados}

Se realiza un análisis de Correlación Factorial, con el programa Amos, del SPSS, encontrándose los siguientes Resultados 
Tabla 2.- Regression Weights: (Group number 1 - Default model)

\begin{tabular}{|c|c|c|c|c|c|c|c|}
\hline & & & $\begin{array}{r}\text { Estimat } \\
\mathrm{e}\end{array}$ & S.E. & C.R. & $\mathrm{P}$ & $\begin{array}{l}\text { Labe } \\
1\end{array}$ \\
\hline $\begin{array}{l}\mathrm{v} 1 \\
2\end{array}$ & $\begin{array}{l}-- \\
-\end{array}$ & Capital_Humano & 1.000 & & & & \\
\hline $\begin{array}{l}\text { v2 } \\
3\end{array}$ & $\begin{array}{l}<- \\
-\end{array}$ & $\begin{array}{l}\text { Proceso_Desarrollo_Empresaria } \\
1\end{array}$ & 1.000 & & & & \\
\hline $\begin{array}{l}\text { v2 } \\
4\end{array}$ & $\begin{array}{l}<- \\
-\end{array}$ & $\begin{array}{l}\text { Proceso_Desarrollo_Empresaria } \\
1\end{array}$ & 1.258 & $\begin{array}{r}.11 \\
6\end{array}$ & $\begin{array}{r}10.86 \\
5\end{array}$ & $\begin{aligned} * * \\
*\end{aligned}$ & \\
\hline $\begin{array}{l}\text { v2 } \\
5\end{array}$ & $\begin{array}{l}<- \\
-\end{array}$ & $\begin{array}{l}\text { Proceso_Desarrollo_Empresaria } \\
1\end{array}$ & 1.243 & $\begin{array}{r}.11 \\
4\end{array}$ & $\begin{array}{r}10.87 \\
0\end{array}$ & $\begin{aligned} * * \\
*\end{aligned}$ & \\
\hline $\begin{array}{l}\text { v1 } \\
3\end{array}$ & $\begin{array}{l}<- \\
-\end{array}$ & Capital_Humano & .717 & $\begin{array}{r}.10 \\
0\end{array}$ & 7.160 & $\begin{array}{r}* * \\
*\end{array}$ & \\
\hline $\begin{array}{l}\text { v1 } \\
4\end{array}$ & $\begin{array}{l}<- \\
-\end{array}$ & Capital_Humano & 1.094 & $\begin{array}{r}.09 \\
4\end{array}$ & $\begin{array}{r}11.65 \\
3\end{array}$ & $\begin{aligned} * * \\
*\end{aligned}$ & \\
\hline $\begin{array}{l}\text { v1 } \\
5\end{array}$ & $\begin{array}{l}<- \\
-\end{array}$ & Capital_Humano & .966 & $\begin{array}{r}.10 \\
0\end{array}$ & 9.709 & $\begin{aligned} * * \\
*\end{aligned}$ & \\
\hline $\begin{array}{l}\text { v1 } \\
6\end{array}$ & $\begin{array}{l}<- \\
-\end{array}$ & Capital_Humano & 1.032 & $\begin{array}{r}.08 \\
7\end{array}$ & $\begin{array}{r}11.87 \\
8\end{array}$ & $\begin{aligned} * * \\
*\end{aligned}$ & \\
\hline $\begin{array}{l}\text { v1 } \\
7\end{array}$ & $\begin{array}{l}<- \\
-\end{array}$ & Capital_Humano & .742 & $\begin{array}{r}.10 \\
3\end{array}$ & 7.188 & $\begin{aligned} * * \\
*\end{aligned}$ & \\
\hline
\end{tabular}

Valores significativos menores a 0.001 (P en la tabla) igual $* * *$

Fuente: Elaboración Propia

En la tabla 2 se encontró que los pesos de la razón crítica (CR) todos sobrepasan el valor de 1.96 lo que señala que son significativas, al nivel de 0.001 como lo muestra la columna de P. lo anterior denota que las variables utilizadas presentan valores significativos (valor Beta) sobre los constructos, señalando con ello que los indicadores son los adecuados en el análisis. 
Figura 1.- Representación Gráfica del Modelo. (Byrne, 1994)

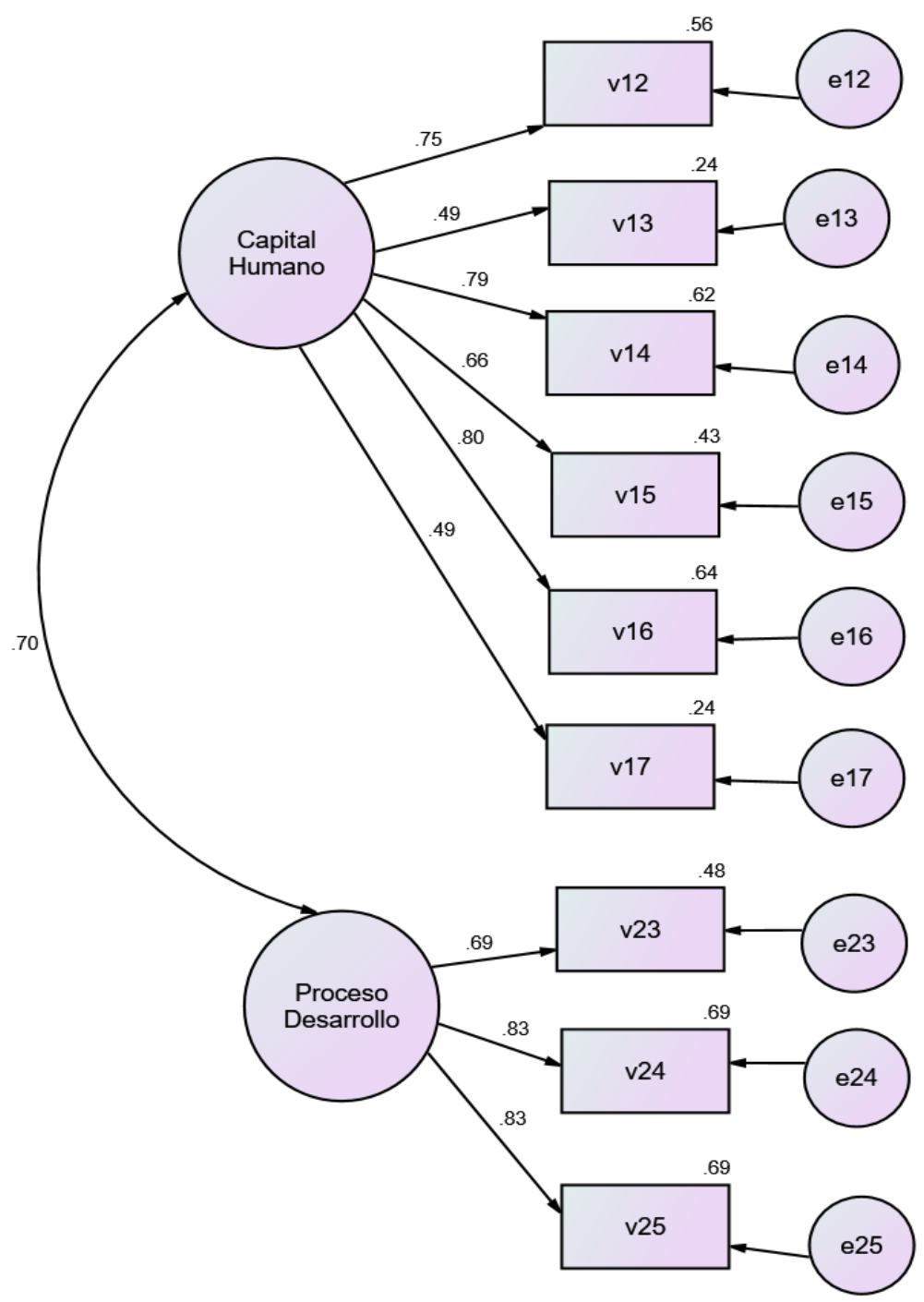

Fuente: Elaboración Propia

La figura 1.- muestra que las variables correspondientes a los dos constructos, Capital Humano y Proceso de Desarrollo Empresarial, presentan una correlación de 0.70 y una varianza Explicada de 49 \%; los valores de los pesos de la regresión lineal múltiple (Betas), de cada una de las variables simples presentan valores por encima de 0.49 , y por debajo de 0.83 ; las correlaciones múltiples se encuentran en un rango 0.24 y 0.69 , lo que señala que la varianza explicada está en valores entre el $40 \%$ y $49 \%$. Esto muestra 
que existe una correlación entre el capital intelectual de los empleados y la realización de los procesos de desarrollo de las empresas de la región sur de Coahuila. Lo que prueba la hipótesis planteada de a mayor gestión de capital Humano, mejores procesos de desarrollo empresarial.

Estos datos proporcionan la Prueba de Hipótesis alterna planteada.

Tabla 3.- Baseline Comparisons

\begin{tabular}{|c|ccccc|}
\hline Model & $\begin{array}{c}\text { NFI } \\
\text { Delta1 }\end{array}$ & $\begin{array}{c}\text { RFI } \\
\text { rho1 }\end{array}$ & $\begin{array}{c}\text { IFI } \\
\text { Delta2 }\end{array}$ & $\begin{array}{c}\text { TLI } \\
\text { rho2 }\end{array}$ & CFI \\
\hline Default model & .954 & .937 & .983 & .976 & .983 \\
Saturated model & 1.000 & & 1.000 & & 1.000 \\
Independence model & .000 & .000 & .000 & .000 & .000 \\
\hline
\end{tabular}

Fuente: Elaboración propia

Los Estadísticos de ajuste se pueden encontrar en la Tabla 2, donde los valores de Índice de Parsimonia NFI; Índice de Ajuste Relativo RFI; Índice de Ajuste Incremental IFI; Coeficiente de Tucker-Lewis TLI, y el Índice de ajuste comparativos CFI se encuentran por encima de valores a .9 lo que señala que el modelo es parsimonioso

Tabla 4.- RMSEA

\begin{tabular}{|c|cccc|}
\hline Model & RMSEA & LO 90 & HI 90 & PCLOSE \\
\hline Default model & .049 & .015 & .076 & .496 \\
Independence model & .314 & .296 & .332 & .000 \\
\hline
\end{tabular}

Fuente: Elaboración propia

En la tabla 4 se encuentran los resultados de la Raíz media cuadrada RMSEA, con un valor de 0.49 menor que el alfa de 0.05 lo que señala un ajuste perfecto de los datos del modelo. Esto muestra que los datos de las variables simples ajustan bien con respecto a los constructos analizados, por lo tanto, las correlaciones y los pesos de los coeficientes parciales estandarizados son significativos.

\section{Conclusiones}

Considerando la importancia que algunos de los estudios citados dan a la aplicación del Capital Humano junto con el Capital Estructural son los principales elementos que permiten la diversificación de los negocios, de acuerdo al contexto, es difícil de separar a las personas de y sus comportamientos de las gestiones que se utilizan en la empresa, debido a que las personas son quienes forman, deciden y actúan, en nombre de las empresas es la gente que labora en ellas.

De acuerdo con la llamada cuarta revolución industrial es necesario comprender que tanto el capital humano como el capital estructural, que es el capital que conforma los bienes tangibles de funcionalidad en las empresas, 
por lo tanto se tienen que considerar que el capital humano debe de tener acceso a las tecnologías de la información y comunicación.

La cuarta revolución industrial que comprende la inclusión de las tecnologías de la información y la comunicación es una fase en la que actualmente se encuentran los empresarios alrededor del mundo, ya que la inclusión de toda la tecnología de punta en cuanto a las comunicaciones y a los manejos de la informática que utilizan técnicas como el análisis de constructos y de grandes cantidades de población es al parecer lo que permite una mejora en los desarrollos empresariales, pero con el ingrediente necesario del Capital Intelectual.

Con estos dos elementos de capital estructural y tecnologías de información y comunicación se puede considerar que el capital humano tiene entonces muchas ventajas para realizar ventajas competitivas que ayudan a que las empresas desarrollen mejores estrategias de competitividad, es decir el capital humano es importante en el proceso de desarrollo de la empresa cualquiera que fuera el giro de la misma. Lo que permite comprobar la Hipótesis alterna planteada.

Considerando que históricamente las empresas y las personas que laboraban en ellas están totalmente antagonistas, se pensaba que los objetivos de las empresas eran contrarios a los objetivos de las personas, se considera esta etapa como algo que estaba obscuro entre el reconocimiento de los objetivos y las soluciones para llegar a ellos.

De acuerdo con los resultados, del presente trabajo, se debe considerar que las variables que se manipularon para establecer los constructos, habla de consideraciones acerca de los salarios, y en cuanto a las características de los mismos pueden establecerse como adecuados, de acuerdo a la percepción de las personas en la Región sur de Coahuila; otra de las variables fueron la consideración de pertenencia a la empresa, la percepción de cómo los ve la empresa en función de los conocimientos, y, si estos son incrementados por parte de los corporativos del negocio.

Además, la ganancia en experiencia de parte de las personas que son el Capital humano dentro de las empresas tiene una importancia definitiva en cuanto al manejo de los procesos de desarrollo de la fábrica, es por esto que, de acuerdo con el planteamiento de la hipótesis generalizada solamente a la Región Sur del estado de Coahuila, se demuestra al señalar que el Capital Humano tiene importancia para el desarrollo de los procesos de toda Empresa.

Un punto importante es establecer que se tiene que cumplir con los cánones establecidos por las reglas de capacitación, ya que esta no es una forma de educación como la tradicional; sino que es una forma específica de aprendizaje que está en función de los objetivos de desarrollo empresarial. En la población donde se llevó a cabo este trabajo de investigación geográficamente es la región suroeste del Estado de Coahuila, por lo que se 
puede sustentar que los procesos utilizados para la capacitación, en verdad contribuyen en altos porcentajes para que las empresas puedan lograr sus objetivos, es decir los procesos de capacitación se encuentran bastante relacionados con el desarrollo empresarial de la región.

\section{References:}

1. Brunner, J. J., \& Gregory., E. (2003). Informe de Capital Humano en Chile . Santiago de Chile : Universidad Adolfo Ibáñez.

2. Byrne, B. M. (1994). Structural Equation Modeling with EQS and EQS Windows (Basic Concepts, Applications, and programming). Thousand, Oaks California: Sage Produccion Editor Diane S. Foster.

3. Cesar, P. (2004). Técnicas de Análisis Multivariante de Datos: Aplicaciones con SPSS. Madrid España: Pearson Educación.

4. Charles, L. H., Torres, G. A., \& Castro, L. D. (2018). Efectos del Capital Social en el Empleo en México. Economía Insitucional, 20(38), 263-283.

5. Chiavenato, I. (2019). Administración de los recursos humanos: El capital de la Organización (8th Edición). México: McGraw-Hill.

6. DeNisi, A., \& Hitt, \&. J. (2003). The know-ledge based approach to sustainable competitive advantage . DeNisi, A, y Hitts, M. (Eds) Managing Knowledge for sustained competitive advantage designing Resourse Management San Francisco CA., 3-336.

7. German-Soto, V., Rodríguez, P. R., \& Escamilla, J. N. (2013). Acumulación y Desigualdad del Capital Humano entre los Estados Mexicanos durante 1960-2008. Paradigma Económico , 5-31.

8. Hair, J. F., Black, W. C., Babin, B. J., \& Anderson, R. E. (2010). Multivariate Data Analysis (Seventh Edition). United States of America: Pearson Prentice Hall.

9. Hair, J. F., Bush , R. P., \& Ortinau, D. J. (2010). investigación de Mercados: en un ambiente de Información digital. México: McGrawHill Educación.

10. Landero, Hernández , R., \& González Ramírez , M. (2007). Estadística con SPSS y Metodología de la Investigación. México: Trillas.

11. Paredes, R., \& Pinto, J. (2009). ¿El fin de la Educacióón Pública en Chile? Santiago UKniversidad Adolfo Ibáñez,, 36(1), 47-66.

12. Passos-Simoncas, E. S., \& Arias-Aragonés. (2017). El Capital Humano como factor de competitividad en la industria hotelera y turística en Cartagena. Panorama Económico, 269-282. 